\title{
Interconnecting Lock-step Synchronous Fault-tolerant Systems based on Voting and Error-Correcting Codes
}

\author{
Thijs Krol \\ University of Twente, Department of Computer Science \\ P.O. Box 217, NL7500 AE Enschede, The Netherlands \\ email: krol@cs.utwente.nl
}

\begin{abstract}
The correctness of the behavior of a fault-tolerant system depends among other things on the correct distribution of the data descending from unreliable I/O devices over the modules of the fault-tolerant system, the so-called input-problem. More generally, a maliciously behaving system, whether it is fault-tolerant or not, should never defeat a correctly functioning faulttolerant system, i.e. a system which does not contain more faulty modules than it is designed to tolerate.

This paper presents a new class of synchronous deterministic non-authenticated algorithms for reaching Byzantine agreement on data descending from other (fault-tolerant) devices. The algorithms are based on voting and error-correcting codes and require considerably less data communication than the existing algorithms, whereas the number of rounds and the number of modules meet the minimum bounds.
\end{abstract}

\section{Introduction}

Fault-tolerant system (ft-system) in practice are always built from a number of different fault-tolerant subsystems, each having different reliability requirements and properties. Such a system could, for example, consist of subsystems that are divided into three distinct reliability levels, viz. the external inputs that are basically unreliable, a reliable reconfigurable redundant storage system and a supervisory service with high reliability. In any case a ft-system will be built from a fault-tolerant part and unreliable inputdevices. Communication between these subsystems must be such that data originating from a malfunctioning subsystem never causes the receiving system to break down. So the interconnection between these ft-subsystems has to be done very carefully.

Without applying special precautions, broadcast faults (Byzantine fault) in which a malfunctioning source sends different data to the various modules of a receiving ft-system, might cause a correctly function- ing $\mathrm{ft}$-receiving system to go down, even if the number of faults in the receiving system is not more than it is designed to tolerate.

In any ft-system the data are replicated, or encoded with some error-correcting code [5], and distributed over a number of modules such that the date can be recovered in case one or more modules fail. Similarly, the processes (functions) which act on this data are replicated and distributed over a number of modules such that the data that result from these processes is again fault resilient. So in a ft-system the data remains recoverable (fault resilient) as long as the number of faulty modules does not exceed a certain number. However this fault resilience depends on the fact that the input data for the replicated processes is fault resilient too. So all correct processes must start from the same data, which is not guaranteed in case of broadcast (Byzantine) faults.

Broadcast faults in the communication between a transmitting and a receiving ft-system, may be caused by power, driver and clock failures. The physical processes behind these failures are slow compared to the data transmission rate and therefore ambiguous data values, which are interpreted differently by the receiving modules, are likely to be sending by the transmitting system.

One should distinguish between the probably incorrect data that is produced by a malfunctioning source and the fact that a malfunctioning source sends different data to the various modules of a fault tolerant system. The application programs should be able to keep up with incorrect date while the communication protocols between the ft-systems must cope with the broadcast faults. The latter will be called the inputproblem. The algorithms that solve the input-problem will be called input-agreement algorithms.

The input-agreement algorithms are related to, but different from the consensus and interactive consistency algorithms. 
With consensus a set of modules is considered, of which at most $T$ are malfunctioning. Each module possesses an initial value $v_{i}$. After completion of the protocol, the correct receiving modules all decide on the same value and if all initial values are equal, say $v$, then all processors decide on the value $v$.

With interactive consistency again a set of modules is considered each possessing an initial value. In this case, after completion of the protocol, all modules agree on the initial value they think they have received from each of the modules. For every module $p$ it holds that if $p$ is correct, the above mentioned agreement equals the initial value actually sent by processor $p$.

An interactive consistency (IAC) algorithm, in which only one source module is considered, is also called a Byzantine agreement algorithm. For detailed classification of consensus and IAC algorithms we refer to [10], chapter 3 and [1].

Opposite to the consensus and IAC algorithms, in which only a single ft-system is considered, the inputagreement algorithms deal with a system consisting of two distinct subsystem, a possibly ft-transmitting system, the t-system, which may be malfunctioning, and a ft-receiving system, the r-system. Notice that a ftsystem is functioning correctly if it contains no more faults than it is designed to tolerate. Input-agreement requires that all correctly functioning modules in the correctly functioning r-system agree on the value they receive from the t-system and if the t-system is functioning correctly this value corresponds to the value which was actually send by the t-system.

The input-problem defined in this way has been addresses for first time in $[4,5]$. In the current paper a new class of input-agreement algorithms is described which includes the ones published in [4], but additionally contains algorithms which require considerably less communication. These algorithms are based on symbol-error-correcting codes and take advantage of the IAC algorithms described in [7]. The algorithms described in this paper require a lock-step synchronous environment and are non-authenticated.

In section 2 we will give a precise description of our system model and a definition of the input-agreement problem. In section 3 a few results of [7] needed in this paper, will be summarized. Thereafter in section 4 and 5 two classes of input-agreement algorithms will be described and its properties will be proven. In section 6 the various algorithms will be compared and discussed.

\section{The system model}

We consider a ft-system consisting of two subsystems, the t-system and the r-system. The t-system trans- mits data to the r-system. The t-system consists of a set $\mathbf{N s}_{t}$ of $n_{t}$ modules $\left(\left|\mathbf{N s}_{t}\right|=n_{t}\right)$ and tolerates up to and including $T_{t}$ faulty modules. Similarly the r-system is characterized by $\mathbf{N s}_{r}, n_{r}$ and $T_{r}$. In case we are dealing with a single non-fault-tolerant t-system $n_{t}=1$ and $T_{t}=0$. We consider only lockstep synchronous non-authenticated systems, so faulttolerance is implemented by masking. Therefore, the $n_{t}$ output values (symbols) of the t-system form a code word of a symbol-error-correcting code consisting of $n_{t}$ symbols that allows the correction of $T_{t}$ erroneous symbols at arbitrary locations, $[5,7]$. If the t-system is an N-modular redundant (NMR) system, this symbolerror-correcting code boils down to a simple repetition code consisting of $n_{t}$ identical symbols. In case of a single non-redundant t-system the encoding function is just a simple identity function.

Let $x$ represent the original data value available in the t-system that has to be transmitted to the r-system. Then each of the $n_{t}$ modules in the rsystem possesses one symbol of the encoded version of $x$. These $n_{t}$ symbols are denoted as a function $m_{t}$ on the modules of the t-system. So $m_{t}\left(i_{t}\right)$ with $i_{t} \in \mathbf{N s}_{t}$ is the symbol possessed by module $i_{t}$ of the t-system. The encoding function is modeled by $\mathcal{T}$ such that $m\left(i_{t}\right)=\mathcal{T}\left(i_{t}\right)(x)$ is the symbol of the encoded version of $x$ which is possessed module $i_{t}$.

Starting from the model described above, we define input-agreement as follows:

Definition 1 Input-agreement is satisfied if:

- In a correctly functioning r-system, i.e. at most $T_{r}$ modules are malfunctioning, the wellfunctioning modules $d_{r}, e_{r}$ always agree with each other on the decoded messages they calculate from the data they received from the t-system, i.e. $\operatorname{dec}\left(d_{r}\right)=\operatorname{dec}\left(e_{r}\right)$.

- If the t-system is functioning correctly, i.e. at most $T_{t}$ modules are malfunctioning, the abovementioned agreement should equal the decoded value $x$ of the data actually sent by the t-system, i.e. $\operatorname{dec}\left(d_{r}\right)=x$.

Without proof we state that input-agreement in a lock-step synchronous non-authenticated environment can only be satisfied if $n_{r} \geq 3 T_{r}+1$.

\section{Interactive consistency algorithms which are based on voting and error- correcting codes}

IAC algorithms are defined on a set Ns of $n$ fully interconnected modules. Provided the number of malfunctioning modules is at most $T$, all correct processors 
decide on the same result and if the source $a$ is correct, this result equals the data actually sent by the source. It has been shown that non-authenticated lock-step synchronous algorithms only can exist if $n \geq 3 T+1$ [9] and require at least $T+1$ rounds of communication [3].

The input-agreement algorithms described in this paper and the way in which they are constructed heavily rely on the IAC algorithms described in [7]. These non-authenticated lock step synchronous IAC algorithms are based on the application of symbol-errorcorrecting codes which considerably reduces the communication between the modules.

Because many IAC algorithms exist having the same properties, classes $\mathcal{A}(T, T+1, a, \mathbf{N s})$ of IAC algorithms have been defined. These classes contain algorithms which tolerate $T$ faulty modules and transmit in $T+1$ communication rounds an initial message from the source $a$ to all modules in the set Ns. Notice that $a \in \mathbf{N s}$ and $|\mathbf{N s}| \geq 3 T+1$. A particular algorithm in a class $\mathcal{A}(T, T+1, a, \mathbf{N s})$ lays down the algorithm in detail.

The $T$-symbol-error-correcting codes used in the algorithms are characterized by the parameters $(n, k, b)$, in which $n$ is the number of symbols in a code word, $k$ is the number of data symbols and $b$ is the number of bits per symbol. These codes do exist if $k \geq 1$, $n-k \geq 2 T, b \geq 1$ and $k \geq 2 \Longrightarrow b \geq \log _{2}(n-1)$, [8]. For efficiency reasons we will always choose $n-k=2 T$, see $[7]$.

In an algorithm from the class $\mathcal{A}(T, T+1, a, \mathbf{N s})$ the original message in the source module $a$ is encoded with a $\left(n_{1}, k_{1}, b_{1}\right)$ code and each of the $n_{1}$ symbols is send to a different module, not being the source module. The receiving module treats the received message in the same way as a source module does and thus encodes the received message (symbol) again, with a code $\left(n_{2}, k_{2}, b_{2}\right)$, and forwards the resulting symbols again to different modules that were not yet visited. In round $T+1$ the received symbols are send unchanged to all modules which did not yet receive the message.

After these $T+1$ communication rounds, in each module a decision-making process of $T+1$ decision rounds is executed. During the decision-making process no communication between the modules takes place. Successively in each module the decoder functions $\left(n_{T}, k_{T}, b_{T}\right)$ to $\left(n_{1}, k_{1}, b_{1}\right)$ are applied on the received and calculated data, which finally yields an estimate of the initial message in the source. A particular algorithm thus is characterized by the sequence of coding and decoding functions applied by the algorithm. Each of them is denoted by the applied code. For example the most simple algorithm with $T=1$ and $N=4$ is characterized by $[3,1,1]_{1} t f 2_{3}\langle 3,1,1\rangle_{3}$, in which $[n, k, b]$ and $\langle n, k, b\rangle$ mean encoding and decoding with a $(n, k, b)$ code respectively, $t$ means transmission and $f 2$ means forwarding each symbol to 2 modules. The number of times an encoding, decoding or forwarding is applied in the system is added in subscript.

A detailed discussion on the construction of these algorithms can be found in $[6,7]$.

\section{Input-agreement algorithms based on post-observation}

Let a fault-tolerant system consist of two subsystems as defined in section 2, the t-system which might be fault-tolerant and the r-system with is fault tolerant. Fault-tolerance is implemented by means of masking redundancy. The t-system transmits data to the rsystem. We do not want such a t-system to cause the receiving t-system to go down, regardless of whether the t-system is functioning correctly or behaving maliciously.

The data in the t-system is available in the form of an $T_{t}$ error-correcting code word, such that each module possesses one symbol of the code word. The basic idea, behind this input-agreement algorithm is that each symbol of this code word is encoded with an $T_{r}$ error-correcting code, after which each symbol of the code word this yields is sent to a different module of the r-system, the input-modules. These input modules relay the received data to all modules of the r-system by means of an IAC algorithm. Hence in all modules of the r-system code words become available, which are the encoded versions of the symbols in the t-system. On these code words the decoder function of the $T_{r}$ error-correcting code is applied. After that the code word of the $T_{t}$ error-correcting code might be decoded. Agreement is obtained from the IAC algorithm and the influence of faulty input modules is masked by the $T_{r}$ error-correcting code.

The preceding construction boils down to the following definition

Definition 2 (Post observation) Let the observation function of the t-system be denoted by $\mathcal{T}^{(-1)}$, then

- In all modules $i_{t}$ of the t-system the messages $m\left(i_{t}\right), m\left(i_{t}\right)=\mathcal{T}\left(i_{t}\right)(x)$, are encoded by means of a $T_{r}$-error-correcting code $\mathcal{W}_{i_{+}}$. Each of the resulting symbols of a code word is sent to a different module $a_{r}$ of the r-system, $a_{r} \in \operatorname{Inp}\left(i_{t}\right)$. A module $a_{r}$ forwards this symbol to all modules of the r-system by means of an interactive consistency algorithm of the class $\mathcal{A}\left(T_{r}, T_{r}+1, a_{r}, \mathbf{N s}_{r}\right)$. 
- In each module $d_{r}$ the interactive consistency algorithms bring forth decisions $\operatorname{dec}\left(\left(i_{t}, a_{r}\right) d_{r}\right)$ about the values $m\left(i_{t}, a_{r}\right)$ received by the modules $a_{r}$ from $i_{t}$. On these decisions for each $i_{t}$, first the decoder function $\mathcal{W}_{i_{t}}^{-1}$ is applied, resulting in decisions dec $\left(\left(i_{t}\right) d_{r}\right)$ and thereafter on the latter decisions, again in each module $d_{r}$, the observation function $\mathcal{T}^{(-1)}$ is applied.

The indication "post-observation" stems from the fact that the observation function $\mathcal{T}$ is applied after the IAC algorithms are applied.

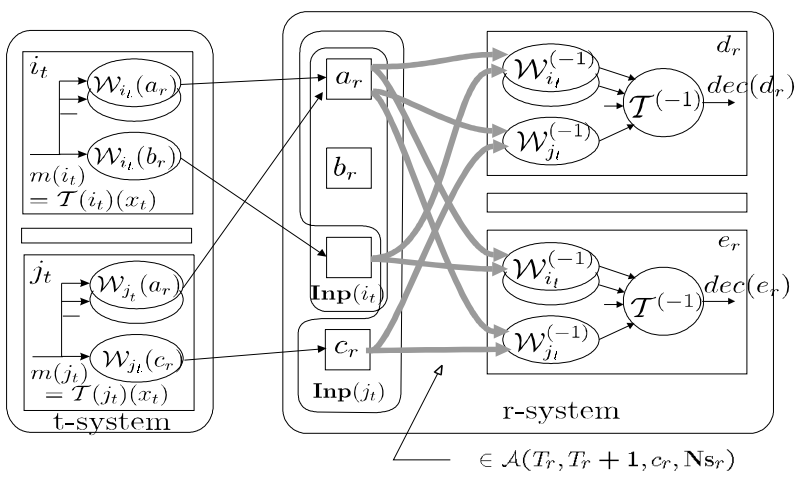

Figure 1: The algorithm based on postobservation

The algorithm is shown in figure 1. In this figure the ft-subsystems and the sets $\operatorname{Inp}\left(i_{t}\right)$ are indicated by boxes with rounded corners, modules by square boxes, direct communications by arrows and function applications by ovals. The communications due to the algorithms from the classes $\mathcal{A}\left(T_{r}, T_{r}+1, a_{r}, \mathbf{N s}_{r}\right)$ are denoted by $\longrightarrow$.

Theorem 1 The input-agreement algorithm based on post-observation defined in definition 2 satisfies the input-agreement properties defined in definition 1.

\section{Proof:}

Let the r-system be functioning correctly, i.e. the number of malfunctioning modules is at most $T_{r}$. The messages $m\left(i_{t}, a_{r}\right)$ received by the r-system from the t-system with $i_{t} \in \mathbf{N s}_{t}$ and $a_{r} \in \operatorname{Inp}\left(i_{t}\right)$, are all forwarded to all modules $d_{r}$ of the r-system by means of an interactive consistency algorithm. Each of these algorithms results in each module $d_{r}$ in a decision $\operatorname{dec}\left(\left(i_{t}, a_{r}\right) d_{r}\right)$ about the value $m\left(i_{t}, a_{r}\right)$. Because the r-system is functioning correctly we obtain from the properties of interactive consistency that for all $i_{t}, a_{r}$ and correct $d_{r}$ and $e_{r}$ holds $\operatorname{dec}\left(\left(i_{t}, a_{r}\right) d_{r}\right)=$ $\operatorname{dec}\left(\left(i_{t}, a_{r}\right) e_{r}\right)$ (agreement). Moreover, because $a_{r}$ is the source module in the IAC algorithm, we know that if $a_{r}$ is correct $\operatorname{dec}\left(\left(i_{t}, a_{r}\right) d_{r}\right)=m\left(i_{t}, a_{r}\right)$.
Let $d_{r}$ and $e_{r}$ be two correctly functioning in the rsystem. The messages $m\left(i_{t}, a_{r}\right)$ received by the modules $a_{r}$, with $a_{r} \in \operatorname{Inp}\left(i_{t}\right)$, from the modules $i_{t}$ are the symbols of the encoded message $m\left(i_{t}\right)$. Hence, if $i_{t}$ and $a_{r}$ are correct then $m\left(i_{t}, a_{r}\right)=\mathcal{W}_{i_{t}}\left(a_{r}\right)\left(m\left(i_{t}\right)\right)$. In the decision making process in each module $d_{r}$, for each $i_{t}$, the decoding function $\mathcal{W}_{i_{t}}^{(-1)}$ is applied on the decisions $\operatorname{dec}\left(\left(i_{t}, a_{r}\right) d_{r}\right)$ with $a_{r} \in \operatorname{Inp}\left(i_{t}\right)$, resulting in decisions $\operatorname{dec}\left(\left(i_{t}\right) d_{r}\right)$. We already concluded that if $a_{r}$ is correct, $\operatorname{dec}\left(\left(i_{t}, a_{r}\right) d_{r}\right)=m\left(i_{t}, a_{r}\right)$. So in case module $i_{t}$ is correct and because at most $T_{r}$ modules $a_{r}$ in $\operatorname{Inp}\left(i_{t}\right)$ are faulty, we obtain $\operatorname{dec}\left(\left(i_{t}\right) d_{r}\right)=m\left(i_{t}\right)$. We know that $m\left(i_{t}\right)=\mathcal{T}\left(i_{t}\right)(x)$, provided $i_{t}$ is functioning correctly and the t-system contains at most $T_{t}$ faulty modules. During the last step of the decision making process, in each module $d_{r}$ of the r-system, the observation function $\mathcal{T}^{(-1)}$ of the t-system is applied on the decisions $\operatorname{dec}\left(\left(i_{t}\right) d_{r}\right)$ with $i_{t} \in \mathbf{N s}_{t}$, bringing forth decisions $\operatorname{dec}\left(d_{r}\right)$. So $\operatorname{dec}\left(d_{r}\right)=x$ if at most $T_{t} \bmod -$ ules of the t-system are malfunctioning. (property 2 of definition 1).

Recall that $\operatorname{dec}\left(\left(i_{t}, a_{r}\right) d_{r}\right)=\operatorname{dec}\left(\left(i_{t}, a_{r}\right) e_{r}\right)$ for any two correct modules $d_{r}$ and $e_{r}$. In all modules the same functions $\mathcal{W}_{i_{t}}^{(-1)}$ and $\mathcal{T}^{(-1)}$ are applied. Hence for the results $\operatorname{dec}\left(d_{r}\right)$ and $\operatorname{dec}\left(e_{r}\right)$ this yield must hold $\operatorname{dec}\left(d_{r}\right)=\operatorname{dec}\left(e_{r}\right),($ property 1 of definition 1$)$

This completes the proof of Theorem 1 .

\section{Input-agreement algorithms based on pre-observation \\ 5.1 Introduction}

The pre-observation method is an efficient alternative to the post-observation method, i.e. it requires less data communication on the account of a little more complex coding scheme.

In the algorithm based on post-observation, The observation function $\mathcal{T}^{(-1)}$ is applied after the IAC algorithms has been applied, (post-observation). By combining the two encoding functions $\mathcal{T}$ and $\mathcal{W}$ into a product code we are able to change the order in which the decoding functions $\mathcal{W}^{(-1)}$ and $\mathcal{T}^{(-1)}$ are applied, in such a way that the observation function $\mathcal{T}^{(-1)}$ is applied immediately after the data has been received by the r-system. That is before the IAC algorithms are applied, (pre-observation).

\subsection{Product codes}

A product code [8] with parameters $\left(n_{t} . n_{w}, k_{t} . k_{w}, b\right)$ is built from two linear codes with parameters $\left(n_{t}, k_{t}, b\right)$ and $\left(n_{w}, k_{w}, b\right)$ respectively. The product code is constructed by arranging the $n_{t} \cdot n_{w}$ symbols of a word of the product code in an $n_{t} \times n_{w}$ matrix such that the columns of the matrix are code words of the $\left(n_{t}, k_{t}, b\right)$ 
code and the rows of the matrix are code words of the $\left(n_{w}, k_{w}, b\right)$ code. The Hamming distance of a product code is the product of the Hamming distances of the underlying codes. This property, however, will not be used. Instead we will use in our input-agreement protocol two other well-known properties of product codes. These properties are expressed in the lemmas 1 and 2 respectively. The proofs can be found in almost any textbook on error correcting codes.

Lemma 1 A code word of an $\left(n_{t} . n_{w}, k_{t} . k_{w}, b\right)$ product code can be constructed from a matrix of $k_{t} \times k_{w}$ data symbols by either first encoding the $k_{w}$ columns with the $\left(n_{t}, k_{t}, b\right)$ code and thereafter encoding the $n_{t}$ rows, this yields, with the $\left(n_{w}, k_{w}, b\right)$ code, or alternatively, by first encoding the rows with the $\left(n_{w}, k_{w}, b\right)$ code and thereafter encoding the resulting $n_{w}$ columns with the $\left(n_{t}, k_{t}, b\right)$ code.

As a consequence, decoding can be applied in any order too.

Lemma 2 An $\left(n_{t} . n_{w}, k_{t} \cdot k_{w}, b\right)$ product code that is constructed from a $T_{t}$-symbol-error-correcting code with parameters $\left(n_{t}, k_{t}, b\right)$ and a $T_{r}$-symbol-errorcorrecting code with parameters $\left(n_{w}, k_{w}, b\right)$ allows the simultaneous correction of $T_{t}$ rows and $T_{r}$ columns of random symbol errors.

In figure 2 the encoding and decoding possibilities of a $\mathcal{T} \times \mathcal{W}$ product code are elucidated. In which $\mathcal{T}$ and $\mathcal{W}$ are an $\left(n_{t}, k_{t}, b\right)$ code and an $\left(n_{w}, k_{w}, b\right)$ code respectively.

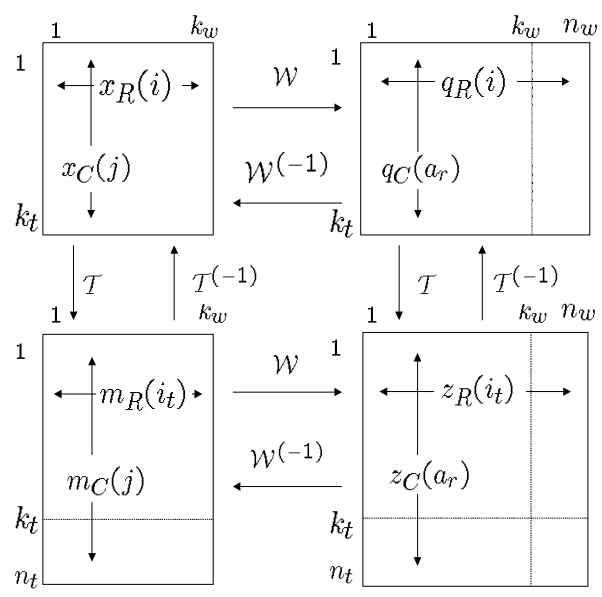

Figure 2: Encoding and decoding sequences in a product code

\subsection{The algorithm}

Again we start from the system model described in section 2, i.e. t-system which transmits data to the fault-tolerant r-system. In both the t-system and the r-system, fault-tolerance is obtained by masking redundancy, such that the data in the t-system can be retrieved with an observation function $\mathcal{T}^{(-1)}$. In this case the initial value $x$ consists of a matrix of $k_{t} . k_{w}$ symbols. The columns of this matrix are encoded by the t-system with the $T_{t}$-error-correcting code $\mathcal{T}$. The algorithm encodes the $n_{w}$ rows with a $T_{r}$-errorcorrecting code $\mathcal{W}$. The word $z$ (an $n_{t} \times n_{w}$ matrix) of the product code $\mathcal{T} \times \mathcal{W}$, this yields, is sent to a set Inp of modules of the r-system, such that a module $a_{r}, a_{r} \in \mathbf{I n p}$ receives a column of $z$, i.e. a code word of $\mathcal{T}$. Each modules $a_{r}$ in Inp decodes this code word and forwards the result to all modules of the r-system by means of an IAC algorithm. On these results the decoding function $\mathcal{W}^{(-1)}$ is applied.

This leads to the following definition and theorem:

Definition 3 Let the initial data value $x$ in the $t$ system consist of $k_{t} . k_{w}$ symbols, i.e. a matrix consisting of $k_{w}$ columns $x_{C}(j)$ of $k_{t}$ symbols and let the data in the t-system be encoded with a $T_{t}$-error-correcting code $\mathcal{T}$, such that each module $i_{t}$ possess $k_{w}$ symbols $m\left(i_{t}, j\right)$ with $m\left(i_{t}, j\right)=\mathcal{T}\left(i_{t}\right)\left(x_{C}(j)\right)$. Furthermore, let Inp be a set of $n_{w}$ modules in the r-system with $2 T_{r}+1 \leq n_{w} \leq n_{r}$ which provides for the communication from the t-system.

- Each module $i_{t}$ in the t-system encodes the word (row) consisting of $k_{w}$ symbols $m\left(i_{t}, j\right)$ with a $T_{r}$ error-correcting code $\mathcal{W}$. The $n_{w}$ symbols $z\left(i_{t}, a_{r}\right)$ with $a_{r} \in \mathbf{I n p}$, this yields, are each transmitted from module $i_{t}$ to module $a_{r}$.

- The modules $a_{r}$ in Inp each have received $n_{t}$ symbols and each apply on the received word of $n_{t}$ symbols the observation function of the T-system $\mathcal{T}^{(-1)}$. Thereafter, each module $a_{r}$ forwards the result of the decoding to all modules of the $r$ system by means of IAC algorithms of the class $\mathcal{A}\left(T_{r}, T_{r}+1, a_{r}, \mathbf{N s}_{r}\right)$.

- In each module of the r-system, the decisions made by the IAC algorithms form a matrix of $n_{w} \times k_{t}$ symbols of which the columns are code words of the code defined by $\mathcal{W}$. The final decision in each module of the r-system is the outcome of decoding these code words.

The algorithm is visualized in figure 3 in the same way as in figure 1 .

A subsystem which is connected to a fault-tolerant subsystem according to the pre-observation method can never cause the latter subsystem to go down, regardless of whether the sending subsystem functions correctly or not, i.e.: 


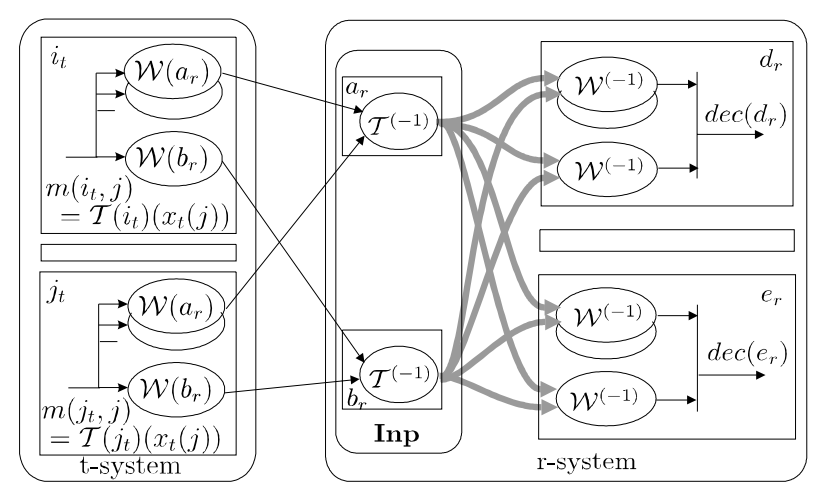

Figure 3: The algorithm based on preobservation

Theorem 2 The Input-agreement algorithm based on pre-observation defined in definition 3 satisfies the input-agreement properties defined in definition 1.

\section{Proof:}

Recall that the original message $x$ is divided into an array of $k_{t} \cdot k_{w}$ symbols $x(i, j)$ of $b$ bits, in which $k_{t}$ and $k_{w}$ are the number of data symbols of the $T_{t}$-errorcorrecting code $\mathcal{T}$ and the $T_{r}$-error-correcting code $\mathcal{W}$ respectively. From the way in which the original message $x$ subsequently is encoded with $\mathcal{T}, \mathcal{W}$ and thereafter transmitted to the modules of the set Inp, we conclude that the $n_{t} . n_{w}$ symbols $z\left(i_{t}, a_{r}\right)$ received by the modules of Inp are code words of the product code $\mathcal{T} \times \mathcal{W}$. In terms of the product code, these symbols form an $n_{t} \times n_{w}$ matrix. A malfunctioning module $i_{t}$ might cause a distorted row in this matrix and a malfunctioning receiving module $a_{r}$ might cause a distorted column. From lemma 2 we know that up to $T_{t}$ rows and $T_{r}$ columns can be corrected. From lemma 2 and 1 we conclude that the order in which the decoding functions $\mathcal{T}^{(-1)}$ and $\mathcal{W}^{(-1)}$ are applied, does not matter. From lemma 1 we know that the $n_{t}$ symbols $z\left(i_{t}, a_{r}\right)$ received by a module $a_{r}$ form a possibly mutilated code word of the code defined by $\mathcal{T}$. So decoding these code words with $\mathcal{T}^{(-1)}$ in each module $a_{r}$, will correct all erroneous symbols caused by malfunctioning processors $i_{t}$ in the t-system, provided the latter contains at most $T_{t}$ faulty modules and provided a module $a_{r}$ in functioning correctly.

The $k_{t}$ symbols obtained in a module $a_{r}$ after decoding with $\mathcal{T}^{(-1)}$ are denoted by $q^{\prime}\left(i, a_{r}\right), 1 \leq i \leq k_{t}$. Hence if the t-system is functioning correctly and $a_{r}$ is correct then, according to lemma $1, q^{\prime}\left(i, a_{r}\right)=q\left(i, a_{r}\right)$ in which for each $i$ the symbols $q\left(i, a_{r}\right)$ are a code word of $\mathcal{W}$ which could be obtained by applying the function $\mathcal{W}$ on the symbols $x(i, j)$ of the original value $x$.

Each module $a_{r}$ communicates the symbols $q^{\prime}\left(i, a_{r}\right)$ with $1 \leq i \leq k_{t}$ to all modules of the r-system by means of an IAC algorithm of the class $\mathcal{A}\left(T_{r}, T_{r}+\right.$ $\left.1, a_{r}, \mathbf{N s}_{r}\right)$. This yields in each correct module $d_{r}$ a number of $k_{t} . n_{w}$ decisions $\operatorname{dec}\left(\left(i, a_{r}\right) d_{r}\right)$ about the symbols $q^{\prime}\left(i, a_{r}\right)$ communicated to $d_{r}$. From the properties of the IAC algorithms we know that if $a_{r}$ is correct $\operatorname{dec}\left(\left(i, a_{r}\right) d_{r}\right)=q^{\prime}\left(i, a_{r}\right)$ and thus if the t-system is also correct $\operatorname{dec}\left(\left(i, a_{r}\right) d_{r}\right)=q\left(i, a_{r}\right)$. So for each $i$ the decisions $\operatorname{dec}\left(\left(i, a_{r}\right) d_{r}\right)$ form a code word of $\mathcal{W}$ containing at most $T_{r}$ erroneous symbols. Decoding these code words with $\mathcal{W}^{(-1)}$ delivers the symbols $x(i, j)$ of the original value $x$.

So if the t-system is functioning correctly in each correct module $d_{r}$, the final decision $\operatorname{dec}\left(d_{r}\right)$ equals the original data value $x$, (property 2 of definition 1 ).

The agreement property of the IAC algorithm guarantees for any correct $d_{r}$ and $e_{r}$ that $\operatorname{dec}\left(\left(i, a_{r}\right) d_{r}\right)=$ $\operatorname{dec}\left(\left(i, a_{r}\right) e_{r}\right)$. In all modules the same function $\mathcal{W}^{(-1)}$ is applied and thus $\operatorname{dec}\left(d_{r}\right)=\operatorname{dec}\left(e_{r}\right)$, (property 1 of definition 1).

This completes the proof of theorem 2 .

\section{Some examples of the interconnec- tion of fault-tolerant systems}

In this section we will elaborate on some system parameters imposed by the input-agreement algorithms. We will present a number of simple examples of the interconnection of fault-tolerant systems by means of input-agreement algorithms with $T_{t}=0$ or 1 and $T_{r}=1$ or 2 . For these examples the number of messages, which needs to be transmitted in order to obtain input-agreement on one message, will be presented and the algorithms based on post- and pre-observation will be compared. The examples will elucidate the way in which the parameters are chosen and the way in which the algorithms are constructed.

Post-observation: (example 8 in table 1)

We interconnect two ft-systems both based on masking according to the $(4,2)$-concept [5]. So $n_{t}=$ $n_{r}=4$ and $T_{t}=T_{r}=1$. The initial data value $x$ in the t-system is encoded with a single-symbolerror-correcting code $\mathcal{T}$, with parameters $\left(n_{t}, k_{t}, b_{t}\right)$. From $n_{t}=4, T_{t}=1, n_{t}-k_{t} \geq 2 . T_{t}$ and $b_{t} \geq$ $\log _{2}\left(n_{t}-1\right.$ ), we obtain $k_{t}=2$ and $b_{t}=2$ (preliminary choice). Each symbol of this code word is encoded with a $T_{r}$ error-correcting code $\mathcal{W}_{i_{+}}$with parameters $\left(n_{w, i_{t}}, k_{w, i_{t}}, b_{w, i_{t}}\right)$, in which $i_{t}$ is the module in the tsystem. Choosing different codes for different $i_{t}$ has no advantage. For efficiency reasons we choose the sets $\operatorname{Inp}\left(i_{t}\right)$ as large as possible. So $\operatorname{Inp}\left(i_{t}\right)=\mathbf{N s}_{r}$ and thus a $(4,2,2)$ code $\mathcal{W}$ suffices. The data word of this 
code consists of two symbols of two bits, so these four bits must correspond to a symbol of the code $\mathcal{T}$. Hence $\mathcal{T}$ must be a $(4,2,4)$ code. For a general method for determining the symbol size we refer to $[7,6]$.

In total 16 symbols of 2 bits are transferred from the t-system to the r-system for communicating 2 data symbols of 4 bits. So per source messages an amount of 4 messages are transmitted.

The four symbols received by each module $a_{r}$ in $\mathbf{N s}_{r}$ from the four modules of the t-system are communicated to all modules of the r-system by means of algorithms of the class $\mathcal{A}\left(1,2, a_{r}, \mathbf{N s}_{r}\right)$. This class contains only one algorithm, which is defined as follows: The message in $a_{r}$ is encoded with a $(3,1,1)$ code, $\mathcal{B}$. Each of the symbols of the code word is sent to a different module. In total $3 \times 4 \times 4$ symbols of 2 bit are communicated in this round. During the last round the 48 symbols are forwarded unchanged to all modules (exactly 2) of the r-system which did not yet receive the message. This gives 96 communication. So in total 144 symbols of 2 bits are communicated inside the r-system for communicating 2 data symbols of 4 bits. Per source messages in the t-system, this requires 36 messages to be transmitted inside the r-system.

During the decision making process in each module $d_{r}$ first the decoder function $\mathcal{B}^{(-1)}$ (majority vote) is applied on the messages received from each module $a_{r}$ (twelve times in each module). Thereafter the decoder function $\mathcal{W}^{(-1)}$ is applied in order to compensate for faulty modules $a_{r}$ (four times in each module) and finally the decoder function $\mathcal{T}^{(-1)}$ is applied (only once in each module).

The algorithm is characterized by the codes that are used successively for communicating the initial data in the t-system to all modules of the r-system. For the algorithm in this example this sequence is $[4,2,4]_{1}[4,2,2]_{4} t\left([3,1,2]_{16} t f 2_{48}\langle 3,1,1\rangle_{48}\right)\langle 4,2,2\rangle_{16}\langle 4,2,4\rangle_{4}$. In which $[n, k, b]$ means encoding with a $(n, k, b)$ code, $t$ means transmission of each symbol of a code word to a different module, $f x$ forwarding unchanged to the remaining $x$ modules and $\langle n, k, b\rangle$ means decoding with a $(n, k, b)$ code. For each encoding and decoding function the number of times the function is applied in the entire system is given in subscript. The sequence of coding and decoding which applies to the IAC algorithm is put between brackets ().

Pre-observation: (example 10 in table 1 )

In this example the same input-agreement problem as in the previous example will be solved with an algorithm based on pre-observation (section 5). So $n_{t}=n_{r}=4$ and $T_{t}=T_{r}=1$. We choose $\mathbf{I n p}=\mathbf{N} \mathbf{s}_{r}$ for efficiency. So the product code is built from a
$(4,2,2)$ code $\mathcal{T}$ and $(4,2,2)$ code $\mathcal{W}$. The initial source message $x$ thus consists of a $2 \times 2$ array of 2 bitsymbols, i.e. 8 bits. After encoding with $\mathcal{W}$, each module $i_{t}$ contains 4 symbols of two bit. This yields a total communication to the r-system of 16 symbols of 2 bits, or an amount 4 messages per source message.

In each modules $a_{r}$ of Inp the decoder function $\mathcal{T}^{(-1)}$ is applied on the received 4 symbols, which results in two symbols of two bits. The latter two symbols are communicated to all modules of the r-system by means of an algorithm of the class $\mathcal{A}\left(1,2, a_{r}, \mathbf{N s}_{r}\right)$. From the previous example and [7] we know that this takes 9 messages per message in $a_{r}$. So in total $4 \times 2 \times 2 \times 9=144$ bits are communicated or an amount of 18 messages per source message.

The algorithm is characterized with the sequence: $[4,2,2]_{2}[4,2,2]_{4} t\langle 4,2,2\rangle_{4}\left([3,1,2]_{8} t, f 2_{24}\langle 3,1,2\rangle_{24}\right)\langle 4,2,2\rangle_{8}$.

The input-agreement algorithm based on preobservation thus only needs 22 messages to be transmitted for one source message, while the algorithm based on post-observation required 40 messages for one source message.

In table 1 some more examples of input-agreement algorithms based on both post- and pre-observation are presented for varying parameters $n_{t}, T_{t}, n_{r}$ and $T_{r}$. In this table m.size denotes the minimum size of the initial value in terms of bits, \#mess $t->r$ gives the amount of data transmitted from the t-system to the r-system in terms of the size of the initial value. Similarly \#mess $r->r$ gives the amount of data transmitted inside the r-system per round of data exchange due to the IAC algorithms and \#mess total the total amount of data transmission.

\section{Discussion}

The problem of interconnecting fault-tolerant lockstep synchronous systems is primarily characterized by the way fault-tolerance is implemented in the t-system and in the r-systems, i.e. by the parameters $n_{t}, T_{t}, n_{r}, T_{r}$ and the observation function $\mathcal{T}$. Comparing the examples in table 1 we see that for the same parameters and $T_{t}>1$, the algorithms based on pre-observation are always superior in terms of communication.

For a single input device there is no difference between pre- and post-observation. Viz., in a non-faulttolerant t-system, i.e. a single system, the data may be considered as being encoded with an identity function. Hence $\mathcal{T}$ is a $(1,1, b)$ code. Clearly the decoding function $\mathcal{T}^{(-1)}$ is an identity function too. Identity functions of course may be omitted in the sequence of encoding and decoding function and thus the algorithms based on post- and pre-observation are identi- 


\begin{tabular}{|c|c|c|c|c|c|c|c|c|c|c|}
\hline $\begin{array}{l}\text { Ex- } \\
\text { am- } \\
\text { ple }\end{array}$ & & ${ }^{t}{ }^{n}{ }^{n}$ & $n_{r}$ & $T_{r}$ & $\begin{array}{l}\text { me- } \\
\text { thod }\end{array}$ & Coding and decoding sequence & $\begin{array}{l}\text { m. } \\
\text { size }\end{array}$ & $\begin{array}{l}\# \text { mess } \\
t->r \\
\text { round } 1\end{array}$ & $\begin{array}{c}\text { \#mess } \\
r->r \\
\text { rnd } 2+3(+4)\end{array}$ & $\begin{array}{c}\# \text { mess } \\
\text { total }\end{array}$ \\
\hline 1 & 1 & 0 & 41 & 1 & post & {$[1,1,1]_{1}[3,1,1]_{1} t\left([3,1,1]_{3} t f 2_{9}\langle 3,1,1\rangle_{9}\right)\langle 3,1,1\rangle_{4}\langle 1,1,1\rangle_{4}$} & 1 & 3 & $9+18$ & 30 \\
\hline 2 & 1 & 0 & 41 & 1 & post & {$[1,1,4]_{1}[4,2,2]_{1} t\left([3,1,2]_{4} t f 2_{12}\langle 3,1,2\rangle_{12}\right)\langle 4,2,2\rangle_{4}\langle 1,1,4\rangle_{4}$} & 4 & 2 & $6+12$ & 20 \\
\hline 3 & 1 & 0 & 41 & 1 & pre & {$[1,1,2]_{2}[4,2,2]_{1} t\langle 1,1,2\rangle_{4}\left([3,1,2]_{4} t f 2_{12}\langle 3,1,2\rangle_{12}\right)\langle 4,2,2\rangle_{4}$} & 4 & 2 & $6+12$ & 20 \\
\hline 4 & 3 & 1 & 41 & 1 & post & {$[3,1,1]_{1}[3,1,1]_{3} t\left([3,1,2]_{9} t f 2_{27}\langle 3,1,2\rangle_{27}\right)\langle 3,1,1\rangle_{12}\langle 3,1,1\rangle_{4}$} & 1 & 9 & $27+54$ & 90 \\
\hline 5 & 3 & 1 & 41 & 1 & post & {$[3,1,4]_{1}[4,2,2]_{3} t\left([3,1,2]_{12} t f 2_{36}\langle 3,1,2\rangle_{36}\right)\langle 4,2,2\rangle_{12}\langle 3,1,4\rangle_{4}$} & 4 & 6 & $18+36$ & 60 \\
\hline 6 & 3 & 1 & 41 & 1 & pre & {$[3,1,1]_{1}[3,1,1]_{3} t\langle 3,1,1\rangle_{3}\left([3,1,1]_{3} t f 2_{9}\langle 3,1,1\rangle_{9}\right)\langle 3,1,1\rangle_{4}$} & 1 & 9 & $9+18$ & 36 \\
\hline 7 & 3 & 1 & 41 & 1 & pre & {$[3,1,2]_{2}[4,2,2]_{3} t\langle 3,1,2\rangle_{4}\left([3,1,2]_{4} t f 2_{12}\langle 3,1,2\rangle_{12}\right)\langle 4,2,2\rangle_{4}$} & 4 & 6 & $6+12$ & 24 \\
\hline 8 & 4 & 1 & 41 & 1 & post & {$[4,2,4]_{1}[4,2,2]_{4} t\left([3,1,2]_{16} t f 2_{48}\langle 3,1,2\rangle_{48}\right)\langle 4,2,2\rangle_{16}\langle 4,2,4\rangle_{4}$} & 8 & 4 & $12+24$ & 40 \\
\hline 9 & 4 & 1 & 41 & 1 & pre & {$[4,2,2]_{1}[3,1,2]_{4} t\langle 4,2,2\rangle_{3}\left([3,1,2]_{6} t f 2_{18}\langle 3,1,2\rangle_{18}\right)\langle 3,1,2\rangle_{8}$} & 4 & 6 & $9+18$ & 33 \\
\hline 10 & 4 & 1 & 41 & 1 & pre & {$[4,2,2]_{2}[4,2,2]_{4} t\langle 4,2,2\rangle_{4}\left([3,1,2]_{8} t f 2_{24}\langle 3,1,2\rangle_{24}\right)\langle 4,2,2\rangle_{8}$} & 8 & 4 & $6+12$ & 22 \\
\hline 11 & 1 & 0 & 72 & 2 & post & {$[1,1,18]_{1}[7,3,6]_{1} t\left([6,2,3]_{7}[5,1,3]_{42} t f 4_{210}\right.$} & & & & \\
\hline 12 & 3 & 1 & 72 & 2 & post & $\begin{array}{c}\left.\langle 5,1,3\rangle_{210}\langle 6,2,3\rangle_{42}\right)\langle 7,3,6\rangle_{7}\langle 1,1,18\rangle_{7} \\
{[3,1,18]_{1}[7,3,6]_{3} t\left([6,2,3]_{21}[5,1,3]_{126} t f 4_{630}\right.}\end{array}$ & 18 & $\frac{7}{3}$ & $7+35+140$ & 184.3 \\
\hline 13 & 3 & 1 & 72 & 2 & pre & $\begin{array}{c}\left.\langle 5,1,3\rangle_{630}\langle 6,2,3\rangle_{126}\right)\langle 7,3,6\rangle_{21}\langle 3,1,18\rangle_{7} \\
{[3,1,6]_{3}[7,3,6]_{3} t\langle 3,1,6\rangle_{7}\left([6,2,3]_{7}[5,1,3]_{42} t f 4_{210}\right.}\end{array}$ & 18 & 7 & $21+105+420$ & 553 \\
\hline 14 & 4 & 1 & 72 & 2 & pre & $\begin{array}{c}\left.\langle 5,1,3\rangle_{210}\langle 6,2,3\rangle_{42}\right)\langle 7,3,6\rangle_{7} \\
{[4,2,3]_{3}[7,3,3]_{3} t\langle 4,2,3\rangle_{7}\left([6,2,3]_{7}[5,1,3]_{42} t f 4_{210}\right.}\end{array}$ & 18 & 7 & $7+35+140$ & 189 \\
\hline & & & & & & $\left.\langle 5,1,3\rangle_{210}\langle 6,2,3\rangle_{42}\right)\langle 7,3,3\rangle_{14}$ & 18 & $\frac{28}{6}$ & $7+35+140$ & 186.7 \\
\hline
\end{tabular}

Table 1: Examples of input-agreement protocols based on pre- or post-observation.

cal in case of a single input device.

The classes of input-agreement algorithms based on post- and pre-observation encompass algorithms that have been published earlier, $[4,5]$. In particular the examples 1, 6 and 9 are published earlier in [4]. In [4], the idea behind the input-agreement algorithms was as follows: Each module of the t-system sends its data to $2 T_{r}+1$ modules of the r-system. If the t-system is a fault-tolerant system, the data received by the modules of the r-system is decoded with the observation function $\mathcal{T}^{(-1)}$ of the t-system. Finally the data is distributed to all modules of the r-system by means of an IAC algorithm. Multi-casting data to $n$ modules is the same as encoding with an $(n, 1, b)$ code and thereafter transmitting each symbol of the code word to a different module of the $n$ modules. So the examples in [4] correspond to algorithms based on pre-observation in which for the function $\mathcal{W}$ a $\left(2 T_{r}+\right.$ $1,1, b)$ repetition code is chosen. We have seen that the application of product codes gives more freedom for the choice of $\mathcal{W}$ and thus leads to more efficient algorithms.

\section{References}

[1] M.Barborak, M.Malek, "The consensus Problem in Fault-Tolerant Computing" ACM Computing Surveys, Vol.25, No 2, June 1993.

[2] D.Dolev and H.R.Strong, "Autenticated algorithms for Byzantine agreement" SIAM COMP, Vol.12, No.4, pp.656-666, Nov. 1983.
[3] M.Fischer and N.Lynch, "A lower bound for the time to assure interactive consistency" Inform. Proc. Letters, Vol.14, pp.183-186, 1982.

[4] Th.Krol, W.J.van Gils, "The Input/Output architecture of the $(4,2)$ concept fault-tolerant computer" 15 th Annual Symposium on Fault-Tolerant Computing, pp254-259, Ann Arbor, MI, June, 1985.

[5] Th.Krol, "(N,K) Concept Fault tolerance" IEEE Tr. on Comp., Vol.C35, No 4, April 1986, pp339-349.

[6] Th.Krol, "A Generalization of fault-Tolerance Based on Masking", Ph.D. dissertation, Eindhoven University of Technology, Eindhoven, the Netherlands, Sept. 1991.

[7] Th.Krol, "Interactive Consistency Algorithms based on Voting and Error-correcting Codes" 25-th Annual Symposium on Fault-Tolerant Computing, pp89-98, Pasadena, CA, June, 1995.

[8] F.J.MacWilliams and N.J.A.Sloane, "The Theory of Error-Correcting Codes." Amsterdam, The Netherlands: North Holland, 1978.

[9] M.Pease, R.Shostak, and L.Lamport, "Reaching agreement in the presence of faults" J. Assoc. Comput. Mach., Vol.27, No.2, pp228, 1980.

[10] A.Postma, "Classes of Byzantine Fault-Tolerant Algorithms for Dependable Distributed Systems", Ph.D. dissertation, University of Twente, Enschede, the Netherlands, Febr. 1998. 